\title{
Three-Dimensional Modeling of Aircraft High-Lift Components with Vehicle Sketch Pad
}

\author{
Erik D. Olson* \\ NASA Langley Research Center, Hampton, VA 23681
}

\begin{abstract}
Vehicle Sketch Pad (OpenVSP) is a parametric geometry modeler that has been used extensively for conceptual design studies of aircraft, including studies using higher-order analysis. OpenVSP can model flap and slat surfaces using simple shearing of the airfoil coordinates, which is an appropriate level of complexity for lower-order aerodynamic analysis methods. For three-dimensional analysis, however, there is not a built-in method for defining the high-lift components in OpenVSP in a realistic manner, or for controlling their complex motions in a parametric manner that is intuitive to the designer. This paper seeks instead to utilize OpenVSP's existing capabilities, and establish a set of best practices for modeling high-lift components at a level of complexity suitable for higher-order analysis methods. Techniques are described for modeling the flap and slat components as separate three-dimensional surfaces, and for controlling their motion using simple parameters defined in the local hinge-axis frame of reference. To demonstrate the methodology, an OpenVSP model for the Energy-Efficient Transport (EET) AR12 wind-tunnel model has been created, taking advantage of OpenVSP's Advanced Parameter Linking capability to translate the motions of the high-lift components from the hinge-axis coordinate system to a set of transformations in OpenVSP's frame of reference.
\end{abstract}

\section{Nomenclature}

$\begin{array}{ll}\mathbf{A}, \mathbf{B} & \text { transformation matrices } \\ b(\bar{x}) & \text { constraint function } \\ b & \text { span of the hinge line } \\ c, s, n & \text { local hinge-axis coordinate system } \\ \mathrm{c} & \text { local wing chord } \\ f(\bar{x}) & \text { objective function } \\ g & \text { gap } \\ L & \text { length of hinge axis } \\ o & \text { overlap } \\ p & \text { hinge-line end point } \\ \bar{x} & \text { vector of design variables } \\ x, y, z & \text { base coordinate system } \\ X, Y, Z & \text { translation components of transformation matrix } \\ \alpha, \beta, \gamma & \text { rotation about the } x-, y-\text {, and } z \text {-axes } \\ \eta & \text { fraction length along semispan } \\ \theta & \text { rotation about the hinge axis } \\ \Gamma & \text { dihedral of the hinge line } \\ \delta & \text { relative deflection } \\ \Delta & \text { increment due to flap deployment } \\ \Lambda & \text { sweep of the hinge line } \\ \text { Subscripts/Superscripts } \\ f & \text { flap } \\ \text { in, out } & \text { inboard, outboard }\end{array}$

*Aerospace Engineer, Aeronautics Systems Analysis Branch, Senior Member AIAA. 


$\begin{array}{ll}s & \text { slat } \\ v & \text { vane } \\ 1 & \text { value following coordinate transformation }\end{array}$

\section{Introduction}

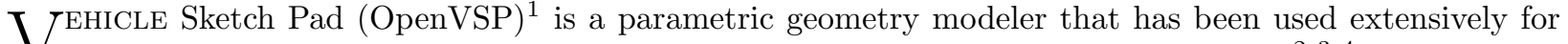
conceptual design studies of aircraft, including studies using higher-order analysis. ${ }^{2,3,4}$ For high-lift configurations, OpenVSP can model flap and slat surfaces using simple shearing of the airfoil coordinates, which is an appropriate level of modeling complexity for lower-order aerodynamic analysis methods. Recent efforts have been successful in using using vortex-lattice based analysis methods to estimate the high-lift aerodynamics of a conventional aircraft model built in OpenVSP. ${ }^{5}$

However, it may be desirable to use a three-dimensional flow solution for unconventional aircraft, such as a blended wing-body or double-bubble fuselage, or for aircraft with low-aspect-ratio or highly-swept wings. For higher-order analysis methods such as panel and Euler codes, the actual three-dimensional surfaces of the high-lift components must be modeled to produce a realistic analysis, particularly for slats and slotted flap systems. Currently there is not a built-in method for defining these components in OpenVSP in a realistic manner, or for controlling their complex motions in a parametric manner that is intuitive to the designer.

To fill this gap, the research described in this paper seeks to establish a set of best practices for modeling high-lift components in OpenVSP at a level of complexity suitable for higher-order analysis methods. Techniques are described for modeling the flap and slat components as separate three-dimensional surfaces, and for controlling their motion using simple parameters defined in the local hinge-axis frame of reference.

\section{Methodology}

The methodology consists of three parts, discussed in each of the following subsections: modeling of the high-lift geometry as separate three-dimensional components in OpenVSP (Section II.A); controlling their motions in a parametric, intuitive manner in order to simulate their deployment during takeoff and landing (Section II.B); and determining the correct deflections and rotations to match published settings in terms of the relative positions of adjacent components ((Section II.C).

\section{A. Modeling of High-Lift Geometry in OpenVSP}

In this methodology, the components of the high-lift configuration-main wing, slat, vane and flap - are modeled in OpenVSP as separate "wing" surfaces, using a planform layout identical to the complete wing; that is, the span, tip chord, root chord, sweep, dihedral and twist for each section of a high-lift component are equal to their associated values from the complete wing. The high-lift surfaces are then turned into subsets of the wing by using airfoil coordinates which are normalized by the chord of the full wing section (Fig. 1). For example, rather than ranging from zero at the leading edge to one at the trailing edge, the abscissas of the $30 \%$-chord flap would range from 0.7 at the leading edge to 1.0 at the trailing edge. In this manner, the span and chord of the high-lift components maintain a parametric link to the complete wing surface, so that modifications to the complete wing surface can easily be applied to the associated high-lift components.

Since OpenVSP does not allow discontinuities in the cross sections, additional short transition segments must be inserted into the main wing surface to allow for a nearly-discontinuous change in cross section between segments with and without flaps (Fig. 2). This allows the main component to be modeled as a continuous surface with only a minor loss of fidelity due to the finite spanwise length of the transition segments.

Unfortunately, using such transition segments has the disadvantage of causing highly-skewed triangles in the exported grid. It is possible to alleviate this problem using the sub-surface tagging ${ }^{6}$ capability in OpenVSP: the transition segments (such as the highlighted transition segment in Fig. 2) can be given unique surface tags in the exported grid to identify those regions for special handling (grid repair, elimination from aerodynamic force calculations, or even elimination from the grid altogether). 


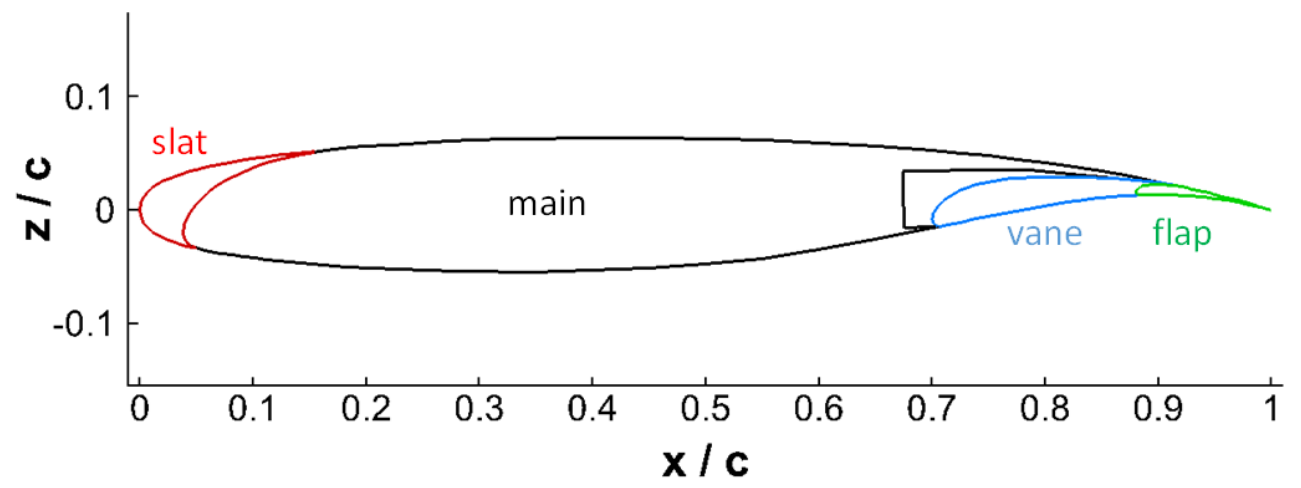

Figure 1. Airfoil coordinates for a wing with a slat and double-slotted flap in the stowed position

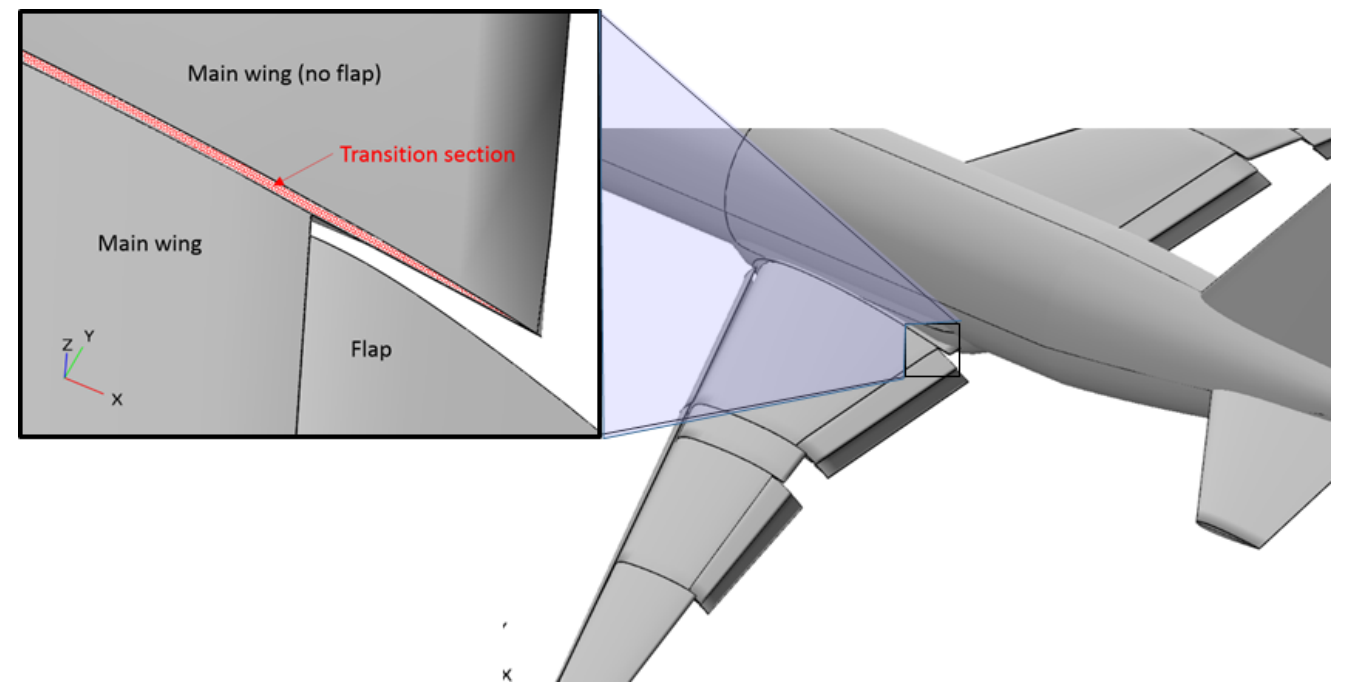

Figure 2. Transition section between flapped and unflapped sections 


\section{B. Controlling Flap and Slat Deflections}

Once the high-lift components have been defined as separate surfaces in the OpenVSP model, it is necessary to define their motion as they are deployed to different flap settings (such as climb, takeoff and landing). OpenVSP allows the user to move a surface by specifying translations in the $x, y$ and $z$ directions and rotations about the $x, y$ and $z$ axes. However, in the case of a flap or slat attached to a wing with sweep, dihedral, and twist, these motions relative to the $(x, y, z)$ axis system are not aligned with the component's hinge axis. It is instead more intuitive to specify the motion as a translation and rotation of an arbitrarilyoriented hinge axis to which the component is attached. In this section, expressions are derived for the rotation and translation of the flap object in the OpenVSP coordinate system that will produce a motion equivalent to the translation and rotation of the flap relative to the hinge axis.

Consider a flap segment with the leading edge of its inboard end located at the origin (Fig. 3). The hinge axis is specified as a line segment of length $L$, passing through $p_{\text {in }}=\left(x_{\text {in }}, 0, z_{\text {in }}\right)$ on the inboard end of the flap and $p_{\text {out }}=\left(x_{\text {out }}, y_{\text {out }}, z_{\text {out }}\right)$ on the outboard end, where

$$
y_{\text {out }}=\sqrt{L^{2}-\left(x_{\text {out }}-x_{\text {in }}\right)^{2}-\left(z_{\text {out }}-z_{\text {in }}\right)^{2}}
$$

Before translation, the hinge axis has the following span, $b$, dihedral, $\Gamma$, and sweep, $\Lambda$ :

$$
\begin{aligned}
b & =\sqrt{y_{\text {out }}^{2}+\left(z_{\text {out }}-z_{\text {in }}\right)^{2}} \\
\Gamma & =\sin ^{-1}\left(\frac{z_{\text {out }}-z_{\text {in }}}{b}\right) \\
\Lambda & =\tan ^{-1}\left(\frac{x_{\text {out }}-x_{\text {in }}}{b}\right)
\end{aligned}
$$

Deployment of the flap is accomplished by a translation of the hinge axis along with its attached flap, followed by a rotation of the flap about the hinge axis.

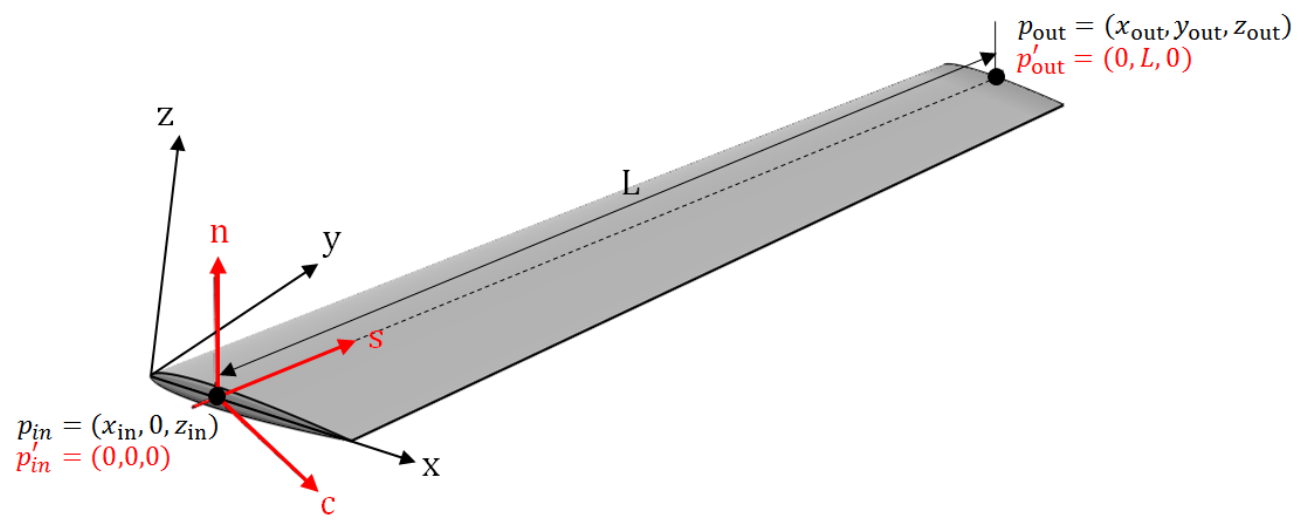

Figure 3. Coordinates for flap geometry

We can specify the translation of the flap relative to a local hinge-axis system, $(c, s, n)$, which has its origin at the inboard end of the hinge axis (Fig. 3): the $c$ axis lies in the $x-y$ plane orthogonal to the hinge axis, the $s$ axis is aligned with the hinge axis, and the $n$ axis is orthogonal to both $c$ and $s$. In this coordinate system, the inboard end of the hinge line is located at $p_{\text {in }}^{\prime}=(0,0,0)$ and the outboard end is located at $p_{\text {out }}^{\prime}=(0, L, 0)$. The inboard end of the hinge line is translated by $\Delta p_{\text {in }}^{\prime}=\left(\Delta c_{\text {in }}, \Delta s_{\text {in }}, \Delta n_{\text {in }}\right)$ and the outboard end by $\Delta p_{\text {out }}^{\prime}=\left(\Delta c_{\text {out }}, \Delta s_{\text {out }}, \Delta n_{\text {out }}\right)$. Since the flap is rigid, the length of the hinge line must remain constant, so

$$
\Delta s_{\text {out }}=\Delta s_{\text {in }}+\sqrt{L^{2}-\left(\Delta c_{\text {out }}-\Delta c_{\text {in }}\right)^{2}-\left(\Delta n_{\text {out }}-\Delta n_{\text {in }}\right)^{2}}-L
$$

Note that the term $\Delta s_{\text {out }}-\Delta s_{\text {in }}$ is always non-positive. This means that if the translations of the inboard and outboard ends are not equal, then the outboard end of the flap will always slide inward along the hinge axis, relative to the inboard end. 
When converted to the $(x, y, z)$ coordinate system, the translation of the inboard end of the flap is

$$
\Delta p_{\text {in }}=\left(\begin{array}{c}
\Delta x_{\text {in }} \\
\Delta y_{\text {in }} \\
\Delta z_{\text {in }}
\end{array}\right)=\left(\begin{array}{c}
\Delta c_{\text {in }} \cos \Lambda+\Delta s_{\text {in }} \sin \Lambda \\
-\Delta c_{\text {in }} \cos \Gamma \sin \Lambda+\Delta s_{\text {in }} \cos \Gamma \cos \Lambda-\Delta n_{\text {in }} \sin \Gamma \\
-\Delta c_{\text {in }} \sin \Gamma \sin \Lambda+\Delta s_{\text {in }} \sin \Gamma \cos \Lambda+\Delta n_{\text {in }} \cos \Gamma
\end{array}\right)
$$

and the translation of the outboard end is

$$
\Delta p_{\text {out }}=\left(\begin{array}{c}
\Delta x_{\text {out }} \\
\Delta y_{\text {out }} \\
\Delta z_{\text {out }}
\end{array}\right)=\left(\begin{array}{c}
\Delta c_{\text {out }} \cos \Lambda+\Delta s_{\text {out }} \sin \Lambda \\
-\Delta c_{\text {out }} \cos \Gamma \sin \Lambda+\Delta s_{\text {out }} \cos \Gamma \cos \Lambda-\Delta n_{\text {out }} \sin \Gamma \\
-\Delta c_{\text {out }} \sin \Gamma \sin \Lambda+\Delta s_{\text {out }} \sin \Gamma \cos \Lambda+\Delta n_{\text {out }} \cos \Gamma
\end{array}\right)
$$

Following translation, the hinge axis's new sweep, $b^{\prime}$, dihedral, $\Gamma^{\prime}$, and sweep, $\Lambda^{\prime}$, are

$$
\begin{aligned}
b^{\prime} & =\sqrt{\left(y_{\text {out }}+\Delta y_{\text {out }}-\Delta y_{\text {in }}\right)^{2}+\left(z_{\text {out }}+\Delta z_{\text {out }}-z_{\text {in }}-\Delta z_{\text {in }}\right)^{2}} \\
\Gamma^{\prime} & =\sin ^{-1} \frac{z_{\text {out }}+\Delta z_{\text {out }}-z_{\text {in }}-\Delta z_{\text {in }}}{b^{\prime}} \\
\Lambda^{\prime} & =\tan ^{-1} \frac{x_{\text {out }}+\Delta x_{\text {out }}-x_{\text {in }}-\Delta x_{\text {in }}}{b^{\prime}}
\end{aligned}
$$

Finally, the flap is rotated about the new hinge axis location by the specified angle, $\theta_{f}$.

In order to express these motions in the OpenVSP frame of reference, we can formulate them in terms of a transformation matrix in homogeneous coordinates. The motion can be broken down into the following sequence of individual transformations:

1. Beginning with the flap in the un-deployed position, translate the flap so that the inboard end of the original hinge axis coincides with the flap origin.

2. Rotate the flap about the $x$-axis by the negative of the flap rotation axis's dihedral angle so that the original hinge axis lies in the $z=0$ plane.

3. Rotate the flap about the $z$-axis by the flap hinge axis's sweep angle so that the original hinge axis coincides with the $y$-axis.

4. Rotate the flap about $y$-axis by the flap rotation angle.

5. Rotate the flap about the $z$-axis by the negative of the new hinge axis's sweep angle.

6. Rotate the flap about the $x$-axis by the new hinge axis's dihedral angle:

7. Translate the flap so that the inboard end of the hinge axis coincides with its new location.

The combined effect of these individual motions can be represented by an overall transformation matrix, A:

$$
\mathbf{A}=\left[\begin{array}{cccc}
A_{11} & A_{12} & A_{13} & X \\
A_{21} & A_{22} & A_{23} & Y \\
A_{31} & A_{32} & A_{33} & Z \\
0 & 0 & 0 & 1
\end{array}\right]
$$


where

$$
\begin{aligned}
& A_{11}=\cos \theta_{f} \cos \Lambda \cos \Lambda^{\prime}+\sin \Lambda \sin \Lambda^{\prime} \\
& A_{12}=-\sin \Gamma \sin \theta_{f} \cos \Lambda^{\prime}-\cos \Gamma \cos \theta_{f} \sin \Lambda \cos \Lambda^{\prime}+\cos \Gamma \cos \Lambda \sin \Lambda^{\prime} \\
& A_{13}=\cos \Gamma \sin \theta_{f} \cos \Lambda^{\prime}-\sin \Gamma \cos \theta_{f} \sin \Lambda \cos \Lambda^{\prime}+\sin \Gamma \cos \Lambda \sin \Lambda^{\prime} \\
& A_{21}=\sin \Lambda \cos \Lambda^{\prime} \cos \Gamma^{\prime}+\cos \Lambda\left(\sin \theta_{f} \sin \Gamma^{\prime}-\cos \theta_{f} \sin \Lambda^{\prime} \cos \Gamma^{\prime}\right) \\
& A_{22}=\cos \Gamma \cos \Lambda \cos \Lambda^{\prime} \cos \Gamma^{\prime}+\sin \Gamma\left(\sin \theta_{f} \sin \Lambda^{\prime} \cos \Lambda^{\prime}+\cos \theta_{f} \sin \Gamma^{\prime}\right) \\
& -\cos \Gamma \sin \Lambda\left(\sin \theta_{f} \sin \Gamma^{\prime}-\cos \theta_{f} \sin \Lambda^{\prime} \cos \Gamma^{\prime}\right) \\
& A_{23}=\sin \Gamma \cos \Lambda \cos \Lambda^{\prime} \cos \Gamma^{\prime}-\cos \Gamma\left(\sin \theta_{f} \sin \Lambda^{\prime} \cos \Gamma^{\prime}+\cos \theta_{f} \sin \Gamma^{\prime}\right) \\
& -\sin \Gamma \sin \Lambda\left(\sin \theta_{f} \sin \Gamma^{\prime}-\cos \theta_{f} \sin \Lambda^{\prime} \cos \Gamma^{\prime}\right) \\
& A_{31}=\sin \Lambda \cos \Lambda^{\prime} \sin \Gamma^{\prime}-\cos \Lambda\left(\sin \theta_{f} \cos \Gamma^{\prime}+\cos \theta_{f} \sin \Lambda^{\prime} \sin \Gamma^{\prime}\right) \\
& A_{32}=\cos \Gamma \cos \Lambda \cos \Lambda^{\prime} \sin \Gamma^{\prime}+\cos \Gamma \sin \Lambda\left(\sin \theta_{f} \cos \Gamma^{\prime}+\cos \theta_{f} \sin \Lambda^{\prime} \sin \Gamma^{\prime}\right) \\
& -\sin \Gamma\left(\cos \theta_{f} \cos \Gamma^{\prime}-\sin \theta_{f} \sin \Lambda^{\prime} \sin \Gamma^{\prime}\right) \\
& A_{33}=\sin \Gamma \cos \Lambda \cos \Lambda^{\prime} \sin \Gamma^{\prime}+\sin \Lambda\left(\sin \theta_{f} \cos \Gamma^{\prime}+\cos \theta_{f} \sin \Lambda^{\prime} \sin \Gamma^{\prime}\right) \\
& +\cos \Gamma\left(\cos \theta_{f} \cos \Gamma^{\prime}-\sin \theta_{f} \sin \Lambda^{\prime} \sin \Gamma^{\prime}\right) \\
& X=x_{\mathrm{in}}+\Delta x_{\mathrm{in}}-\cos \Gamma \sin \theta_{f} \cos \Lambda^{\prime} z_{\mathrm{in}}-\sin \Lambda^{\prime}\left(\sin \Lambda x_{\mathrm{in}}+\sin \Gamma \cos \Lambda z_{\mathrm{in}}\right) \\
& +\cos \theta_{f} \cos \Lambda^{\prime}\left(\sin \Gamma \sin \Lambda z_{\text {in }}-\cos \Lambda x_{\text {in }}\right) \\
& Y=\Delta y_{\text {in }}+\cos \Gamma\left(\sin \theta_{f} \sin \Lambda^{\prime} \cos \Gamma^{\prime}+\cos \theta_{f} \sin \Gamma^{\prime}\right) z_{\text {in }} \\
& -\cos \Lambda^{\prime} \cos \Gamma^{\prime} \cos \Gamma^{\prime}\left(\sin \Lambda x_{\text {in }}+\sin \Gamma \cos \Lambda z_{\text {in }}\right) \\
& +\left(\sin \theta_{f} \sin \Gamma^{\prime}-\cos \theta_{f} \sin \Lambda^{\prime} \cos \Gamma^{\prime}\right)\left(\sin \Gamma \sin \Lambda z_{\mathrm{in}}-\cos \Lambda x_{\mathrm{in}}\right) \\
& Z=z_{\text {in }}+\Delta z_{\text {in }}-\cos \Gamma\left(\cos \theta_{f} \cos \Gamma^{\prime}-\sin \theta_{f} \sin \Lambda^{\prime} \sin \Gamma^{\prime}\right) z_{\text {in }} \\
& -\cos \Lambda^{\prime} \sin \Gamma^{\prime}\left(\sin \Lambda x_{\text {in }}+\sin \Gamma \cos \Lambda z_{\text {in }}\right) \\
& -\left(\sin \theta_{f} \cos \Gamma^{\prime}+\cos \theta_{f} \sin \Lambda^{\prime} \sin \Gamma^{\prime}\right)\left(\sin \Gamma \sin \Lambda z_{\mathrm{in}}-\cos \Lambda x_{\mathrm{in}}\right)
\end{aligned}
$$

Instead of using the hinge-axis coordinates system, however, transformation of a surface in OpenVSP is specified as a rotation around the local $z$-axis by angle $\gamma$, followed by a rotation around the $y$-axis by angle $\beta$, followed by a rotation around the $x$-axis by angle $\alpha$, and finally a translation along the vector $(\Delta x, \Delta y, \Delta z)$. The combined effect of these transformations can be represented by another overall transformation matrix, B:

$$
\mathbf{B}=\left[\begin{array}{cccc}
\cos \beta \cos \gamma & -\cos \beta \sin \gamma & \sin \beta & \Delta x \\
\sin \alpha \sin \beta \cos \gamma+\cos \alpha \sin \gamma & \cos \alpha \cos \gamma-\sin \alpha \sin \beta \sin \gamma & -\sin \alpha \cos \beta & \Delta y \\
\sin \alpha \sin \gamma-\cos \alpha \sin \beta \cos \gamma & \sin \alpha \cos \gamma+\cos \alpha \sin \beta \sin \gamma & \cos \alpha \cos \beta & \Delta z \\
0 & 0 & 0 & 1
\end{array}\right]
$$

If the transformation in the OpenVSP frame of reference, represented by the transformation matrix $\mathbf{B}$, is to be equivalent to the translation and rotation of the hinge axis represented by the transformation matrix $\mathbf{A}$, then it is necessary that $\mathbf{B}=\mathbf{A}$. By process of inspection we can deduce that

$$
\begin{gathered}
\beta=\sin ^{-1} A_{13} \\
\alpha=-\sin ^{-1} \frac{A_{23}}{\cos \beta} \\
\gamma=-\sin ^{-1} \frac{A_{12}}{\cos \beta} \\
\Delta x=X \\
\Delta y=Y \\
\Delta z=Z
\end{gathered}
$$

In other words, if the transformations are specified in OpenVSP using the parameters calculated in Eq. 9, the flap will move according to the transformations originally specified in the $(c, s, n)$ coordinates. 


\section{Matching Gap, Overlap and Relative Deflection}

The previous section showed how the deployment of a high-lift component can be defined in a more intuitive fashion by specifying the motion in the hinge-axis, or $(c, s, n)$, frame of reference. However, published flap positions are typically specified as relative position parameters - gap, overlap, and relative deflection - defined in Fig. 4. The gap is defined as the distance from the lower surface trailing-edge coordinate of the forward component, to the nearest point on the upper surface of the aft component. The deflection and overlap are defined relative to the longest chord of the particular components, which is the distance from the midpoint of the trailing-edge base of the component to the forward-most leading-edge coordinate: the overlap is defined as the distance from the lower-surface trailing edge of the forward component to the forward-most point on the aft component, measured parallel to the longest chord line of the forward component; while the relative deflection ( $\delta$ in the figure) is the angle between the longest chord lines of the forward and aft components.

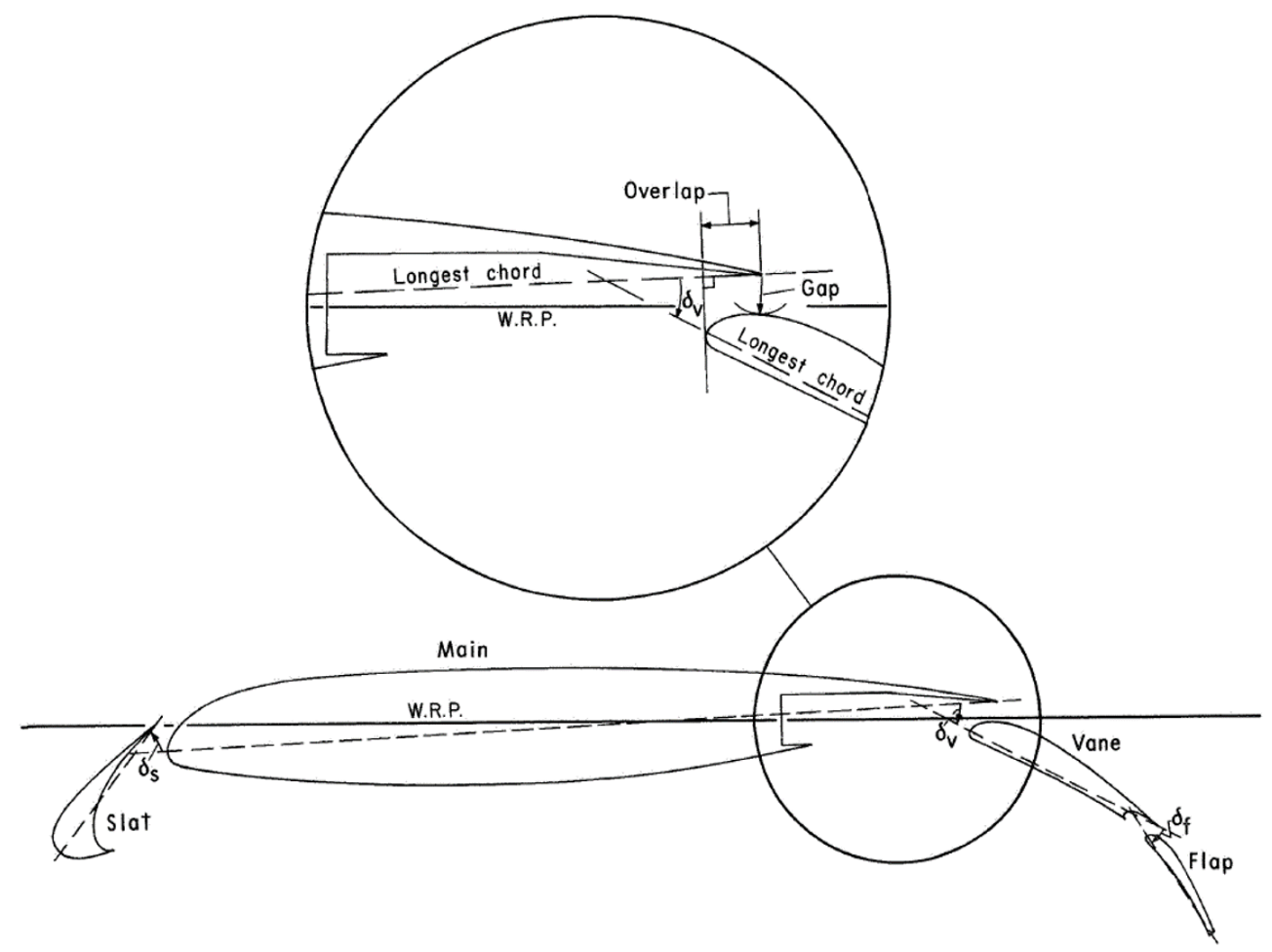

Figure 4. Definition of gap, overlap, and relative deflection $(\delta)$. (Source: Ref. 7)

Since the gap, overlap and relative deflection are indirect measures of the motions of two components, it is difficult to control them directly. If one is trying to match published deflections in terms of these relative position parameters, it becomes necessary to determine the motion in the hinge-axis frame that indirectly results in the desired gap, $g$, overlap, $o$, and relative deflection, $\delta$, for each flap setting. Figure 5 shows how the gap and overlap vary as the hinge axis of a typical flap is translated. The contours of gap and overlap are nearly orthogonal for most of the range of interest, and the overlap is controlled most strongly by the chordwise translation, $\Delta c$, while the gap is controlled most strongly by the vertical translation, $\Delta n$.

One simple method for matching the hinge-axis motions to the desired relative positions is through the use of an optimizer. For each high-lift component, a design-variable vector may be assembled from the inboard and outboard translations and the rotation about the hinge axis: $\bar{x}=\left(\Delta c_{\mathrm{in}}, \Delta n_{\mathrm{in}}, \Delta c_{\mathrm{out}}, \Delta n_{\mathrm{out}}, \theta_{f}\right)$. 


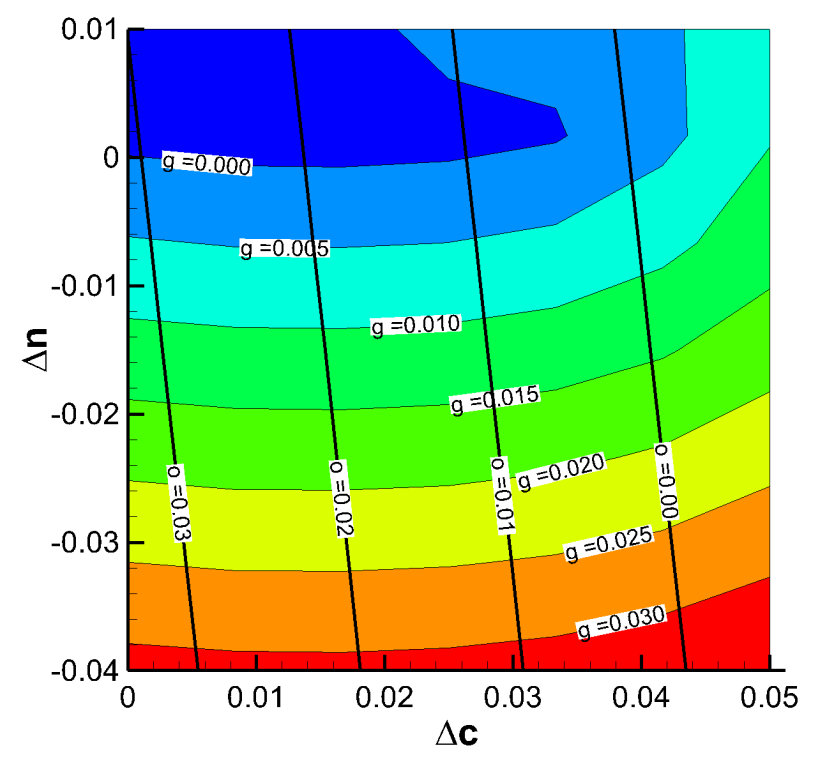

Figure 5. Example variation of gap, $g$, and overlap, o (normalized by chord) with hinge-axis translations in the $c$ and $n$ directions.

The optimizer then varies these parameters in an attempt to minimize the objective function:

$$
\begin{aligned}
& \min _{\bar{x}} f(\bar{x})=b_{g_{\mathrm{in}}}(\bar{x})+6_{g_{\text {out }}}(\bar{x})+6_{o_{\text {in }}}(\bar{x})+b_{o_{\text {out }}}(\bar{x})+b_{\delta}(\bar{x}) \\
& \text { s.t. } \sigma_{g_{\text {in }}}(\bar{x}) \equiv c_{g}\left(g_{\text {in }_{\text {target }}}-g_{\text {in actual }}\right) \geq 0 \\
& \sigma_{g_{\text {out }}}(\bar{x}) \equiv c_{g}\left(g_{\text {out }_{\text {target }}}-g_{\text {out }_{\text {actual }}}\right) \geq 0
\end{aligned}
$$

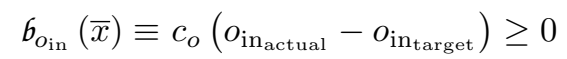

$$
\begin{aligned}
& b_{o_{\text {out }}}(\bar{x}) \equiv c_{o}\left(o_{\text {out }_{\text {actual }}}-o_{\text {out }_{\text {target }}}\right) \geq 0 \\
& b_{\delta}(\bar{x}) \equiv c_{\delta}\left(\delta_{\text {target }}-\delta_{\text {actual }}\right) \geq 0
\end{aligned}
$$

where $c_{g}, c_{o}$, and $c_{\delta}$ are weighting factors in the individual terms. In this formulation it is clear that the optimum is achieved when $f(\bar{x})=0$ and $b_{*}(\bar{x})=0$. This optimization is repeated for each additional segment of the high-lift system until all components are in place.

\section{Example Geometry}

To demonstrate the modeling techniques described in Section II, the Energy Efficient Transport (EET) AR12 wind-tunnel model ${ }^{7,8}$ (Fig. 6) was used as an example. This model is a 12-foot full-span aircraft configuration with a supercritical wing, full-span slats, and part-span double-slotted flaps with a cutout for the engine (Fig. 7).

An OpenVSP model of the EET AR12 was constructed using the published planform shape, airfoil coordinates, twist distribution, and fuselage cross-section shapes. Additional surfaces were created for the main wing component, inboard and outboard slats, inboard and outboard vanes, and inboard and outboard flaps. The slat hinge lines were defined at the slat trailing edge, and the vane and flap hinge lines were defined at each component's leading edge.

The equations for the translations and rotation of each high-lift component were implemented using the Advanced Parameter Linking feature, which allows the user to script complex non-linear relationships between any of the parameters in the model. For each component of the high-lift system, the inboard and outboard hinge axis coordinates $\left(x_{\mathrm{in}}, z_{\mathrm{in}}, x_{\mathrm{out}}\right.$, and $\left.z_{\text {out }}\right)$, the inboard and outboard translations $\left(\Delta c_{\mathrm{in}}, \Delta n_{\mathrm{in}}\right.$, $\Delta c_{\text {out }}$, and $\Delta n_{\text {out }}$ ) and the rotation $\left(\theta_{f}\right)$ were defined using custom user parameters in the model, and the translations and rotation of the component in the OpenVSP frame of reference were calculated using Eq. 9. 


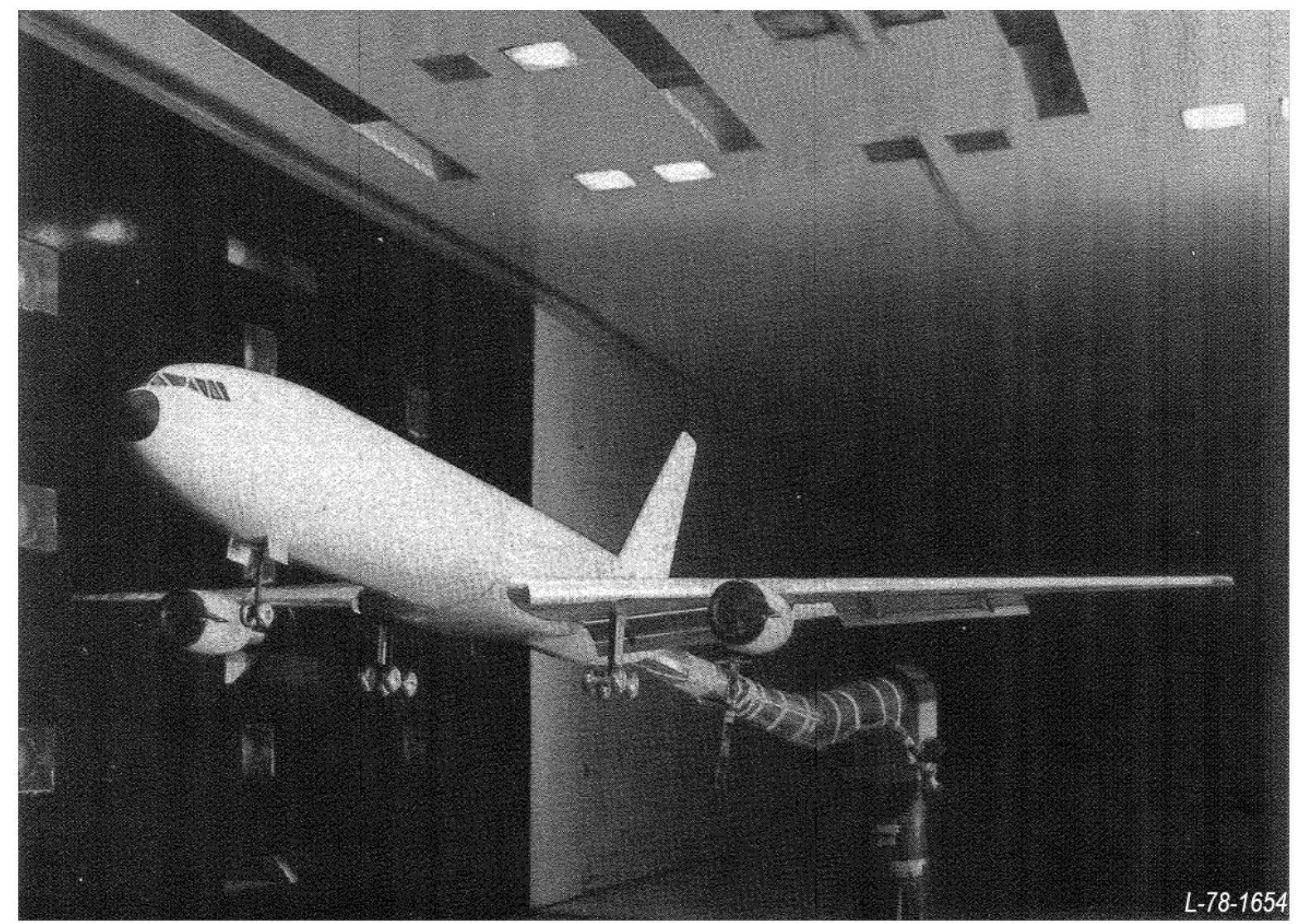

Figure 6. EET AR12 model in the NASA Langley $14 \times 22$ wind tunnel. Source: Ref. 7 

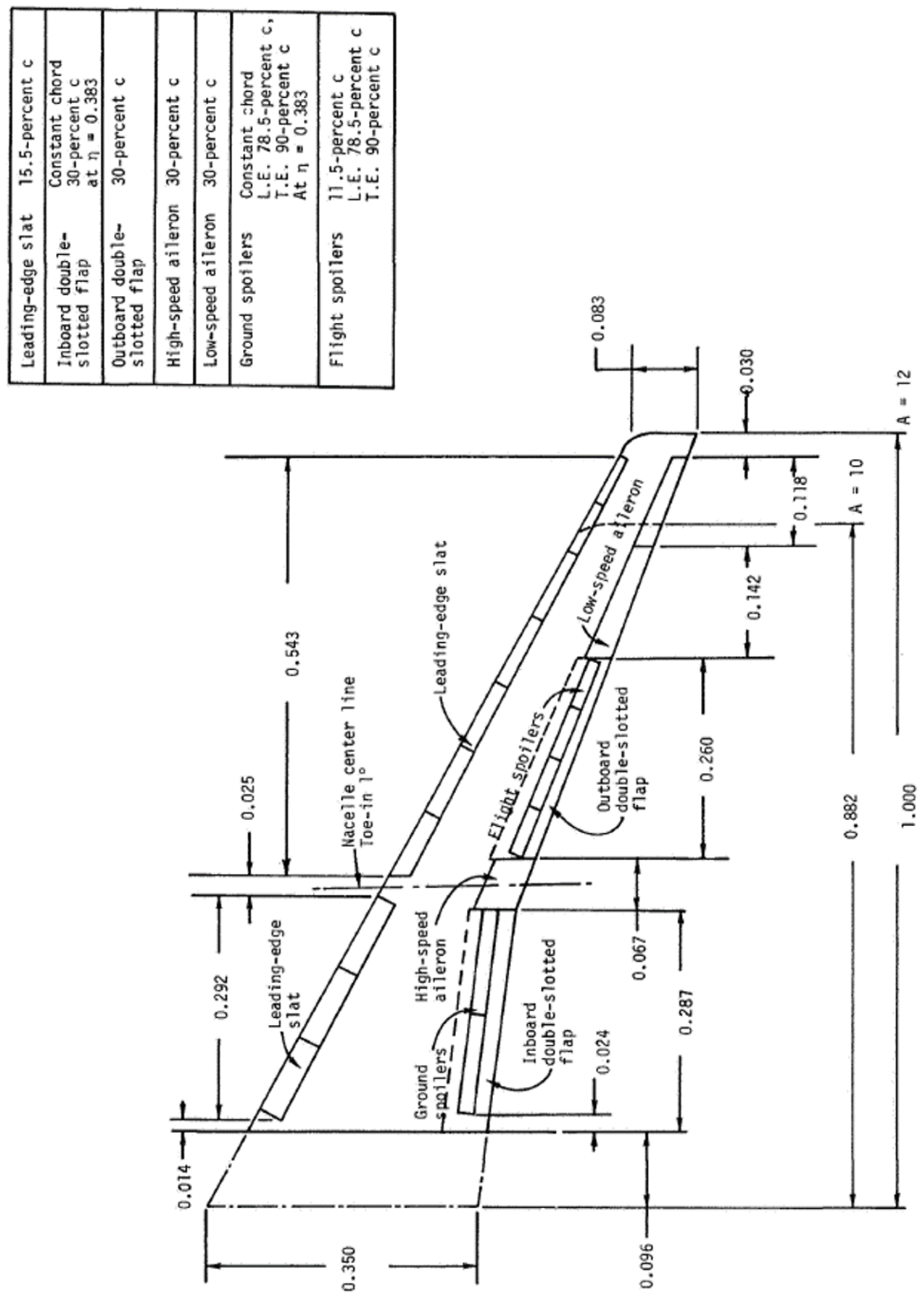

Figure 7. EET AR12 model planform detail. Source: Ref. 7

\section{0 of 14}


Table 1 gives the design gap, overlap and relative deflection of each type of high-lift component for this configuration. The optimization process described in Section II.C was used to determine the translation and rotation of each component that corresponds to these relative positions.

Table 1. Gap, overlap and relative deflection for EET AR12. Source: Ref. 7

\begin{tabular}{|l|l|r|r|r|}
\hline Configuration & Component & Gap $/ c$ & Overlap/c & Rel. Defl., deg \\
\hline Takeoff & Slat & 0.02 & 0.02 & 50 \\
& Vane & 0.015 & 0.04 & 15 \\
& Flap & 0.01 & 0.01 & 15 \\
Landing & Slat & 0.02 & 0.02 & 50 \\
& Vane & 0.02 & 0.03 & 30 \\
& Flap & 0.01 & 0.005 & 30 \\
\hline
\end{tabular}

The results of the optimization process are shown in Fig. 8 for each high-lift component. In each figure, the curves show the path of the inboard and outboard ends of the component's hinge line in the $(c, n)$ plane as the flaps transition from the stowed position to the takeoff setting, and then to the landing setting. The vanes translate the farthest, with the transition taking place in a mostly chordwise direction to bring the vane's leading edge nearly in line with the main component's trailing edge, increasing the effective chord of the section. The flap components, being much smaller than the vanes, have a significantly shorter translation path relative to their stowed position in the vane. Finally, the slat translation occurs in a forward and downward direction, as expected, and the translation distance is proportional to the local chord. Figure 9 shows the final OpenVSP model of the EET AR12 configuration for three cases: the stowed high-lift configuration, the takeoff flap setting, and the landing setting.

\section{Conclusion}

This study establishes a set of best practices for modeling high-lift components in OpenVSP at a level of complexity suitable for higher-order analysis methods, such as panel and Euler codes. The components of the high-lift system are modeled as separate surfaces using OpenVSP's "wing" component, with planform shape parameters equal to their associated values from the complete wing. The components' airfoil coordinates, however, are normalized by the chord of the full wing section to create sections which are subsets of the complete wing section. The motions of each high-lift component as it is deployed are specified in a local hinge-axis coordinate system, which are then converted to the equivalent transformations in the $(x, y, z)$ coordinate system that OpenVSP uses. Finally, an optimization process is described with which the motions of the high-lift components can be determined in order to match published flap deflections, which are typically given in terms of the relative positions of adjacent surfaces (gap, overlap and relative deflection).

The methodology was successfully demonstrated in an OpenVSP model for the EET AR12 wind-tunnel model. The hinge-axis inboard and outboard coordinates, plus the translations and rotations in the hingeaxis frame of reference, were defined using custom user parameters; while the transformation of the highlift component motions into the OpenVSP frame of reference was performed by turning the mathematical relationships derived in this paper into scripted links between the custom user parameters and the built-in OpenVSP transformation parameters.

As an alternative to the scripted parameter linking method, one could take advantage of OpenVSP's custom components by creating a new universal "hinge" component. A flap or slat component could then be attached to the hinge so that its motion would follow that of the hinge. Rather than separate custom user parameters, the motions of the hinge component would be defined as its own properties. An additional advantage to this approach would be that the hinge axis, as an actual component in the model, could be easily rendered graphically in order to visualize its position in the model.

The methodology developed in this paper has already contributed to a study which extends an existing semi-empirical high-lift aerodynamic analysis methodology to include three-dimensional analysis. ${ }^{9}$ It is expected that the current geometric modeling work, as well as the aerodynamic analysis methodology, will eventually become part of a high-lift design and optimization process that will allow designers to investigate the tradeoffs between improved aerodynamic performance and increased system complexity and weight. 


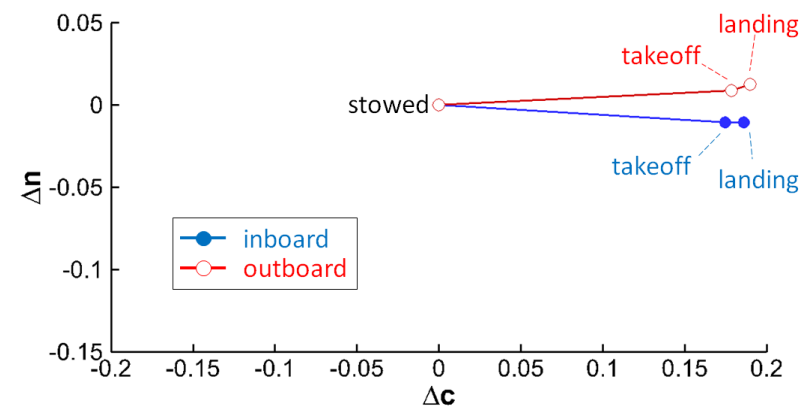

(a) inboard vane

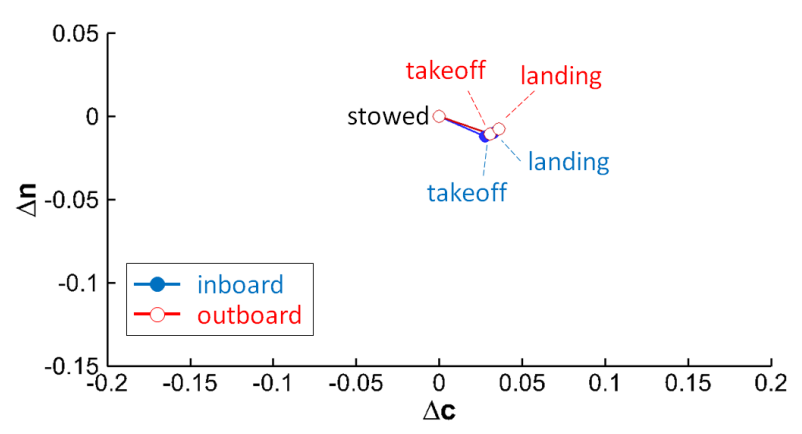

(c) inboard flap

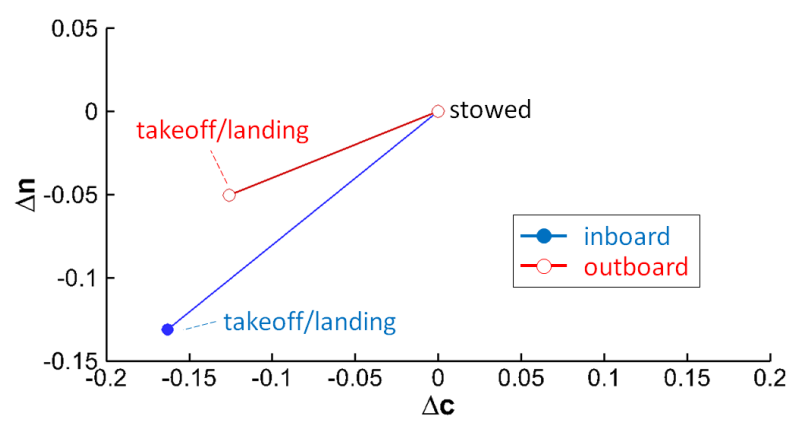

(e) inboard slat

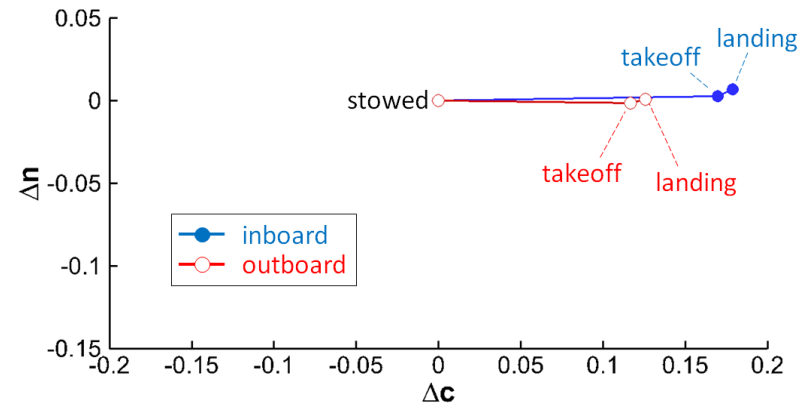

(b) outboard vane

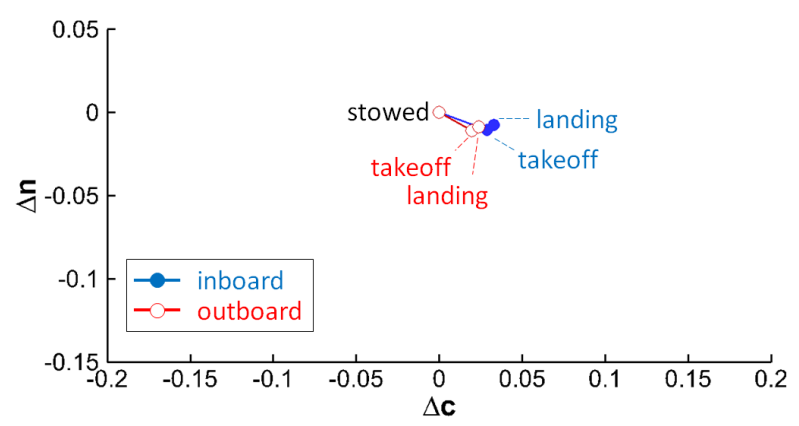

(d) outboard flap

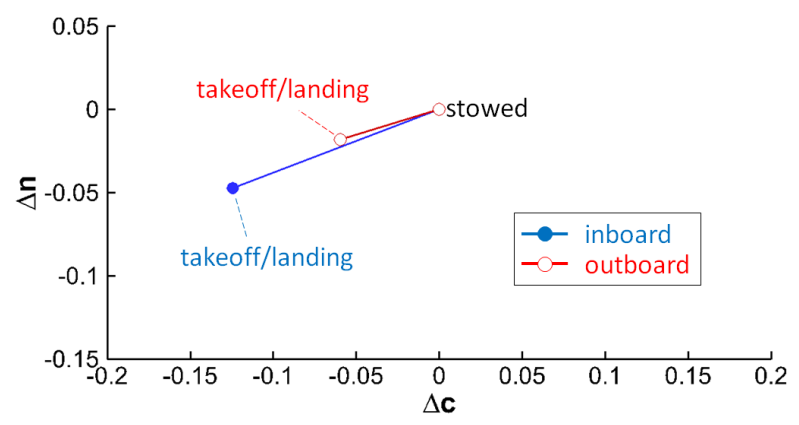

(f) outboard slat

Figure 8. Flap hinge-line translations corresponding to optimized gap and overlap settings for the EET AR12 wing. 


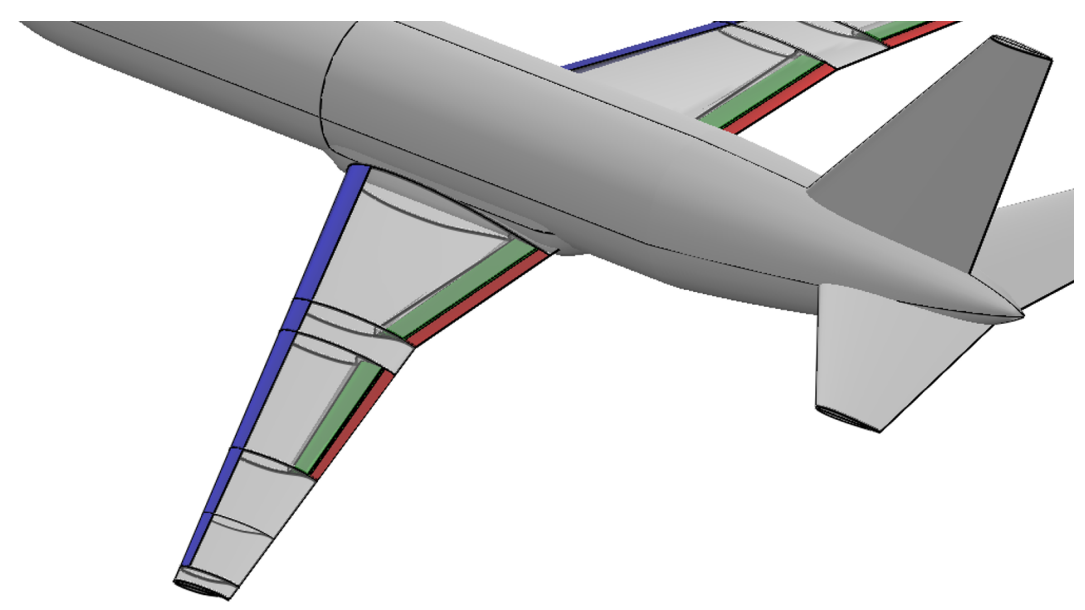

(a) flaps stowed

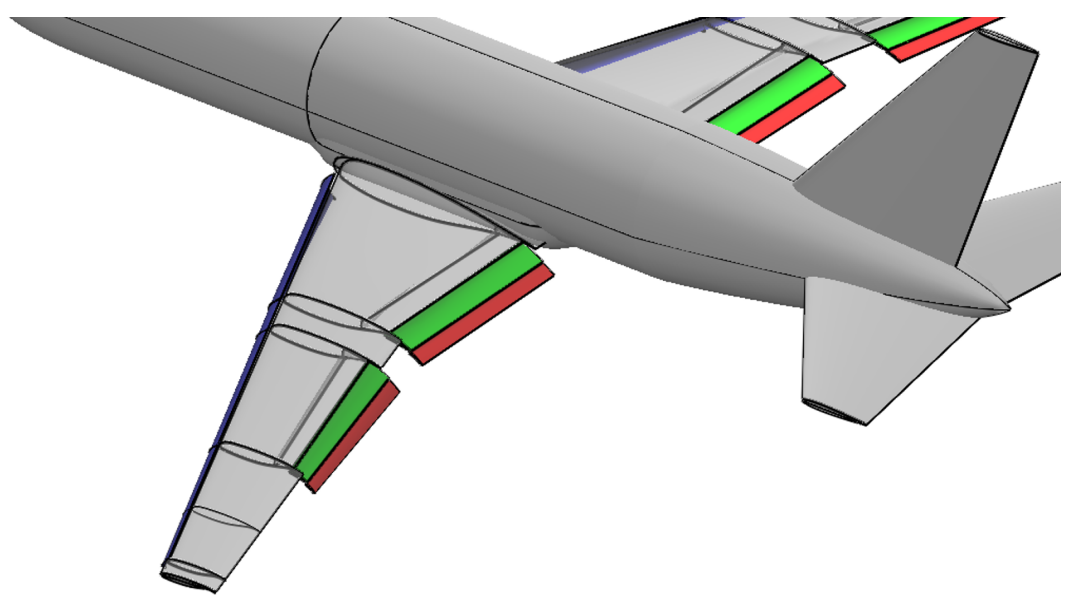

(b) takeoff flaps

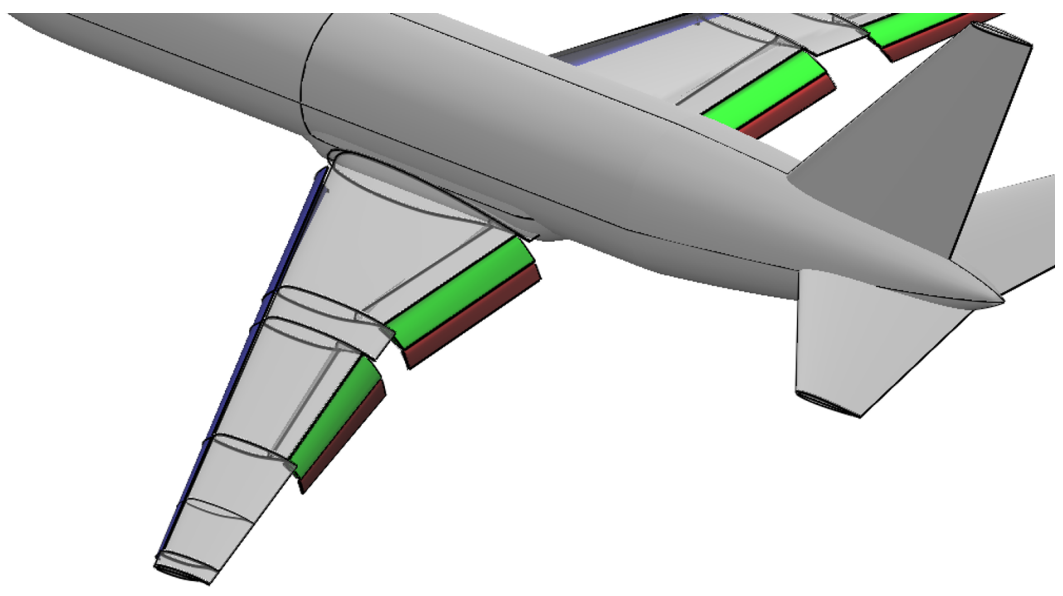

(c) landing flaps

Figure 9. OpenVSP model of the EET AR12 with direct flap modeling. For clarity, the main wing component has been rendered as transparent. 


\section{Acknowledgments}

This work was conducted as part of the NASA Transformational Tools and Technologies Project, led by James D. Heidmann, within the Multi-Disciplinary Design, Analysis and Optimization element, led by Jeffrey K. Viken. The author wishes to thank Andrew Hahn for his editorial assistance.

\section{References}

${ }^{1}$ Hahn, A., "Vehicle Sketch Pad: A Parametric Geometry Modeler for Conceptual Aircraft Design," 48th AIAA Aerospace Sciences Meeting Including the New Horizons Forum and Aerospace Exposition, AIAA 2010-657, Orlando, FL, 2010.

${ }^{2}$ Ordaz, I., "Conversion of Component-Based Point Definition to VSP Model and Higher-Order Meshing," 49th AIAA Aerospace Sciences Meeting including the New Horizons Forum and Aerospace Exposition, AIAA 2011-358, Orlando, FL, 2011.

${ }^{3}$ Chaput, A. and Rizo-Patron, S., "Vehicle Sketch Pad Structural Analysis Module Enhancements for Wing Design," 50th AIAA Aerospace Sciences Meeting, AIAA 2012-0546, Nashville, TN, 2012.

${ }^{4}$ Hahn, A., "Application of Cart3D to Complex Propulsion-Airframe Integration with Vehicle Sketch Pad," 50th AIAA Aerospace Sciences Meeting, AIAA 2012-0547, Nashville, TN, 2012.

${ }^{5}$ Olson, E. D., "Semi-Empirical Prediction of Aircraft Low-Speed Aerodynamic Characteristics," 53rd AIAA Aerospace Sciences Meeting, AIAA, Kissimmee, FL, 2015.

${ }^{6}$ Gary, A. M. and McDonald, R. A., "Parametric Identification of Surface Regions in OpenVSP for Improved Engineering Analysis," 53rd AIAA Aerospace Sciences Meeting, AIAA 2015-1016, Kissimmee, FL, 2015.

${ }^{7}$ Morgan, H. L, Jr. and Paulson, J. W., "Low-Speed Aerodynamic Performance of a High-Aspect-Ratio Supercritical-Wing Transport Model Equipped with Full-Span Slat and Part-Span Double-Slotted Flaps," NASA TP-1580, Dec. 1979.

${ }^{8}$ Morgan, H. L, Jr., "Model Geometry Description and Pressure Distribution Data from Tests of EET High-Lift Research Model Equipped with Full-Span Slat and Part-Span Flaps," NASA TM-80048, Feb. 1979.

${ }^{9}$ Olson, E. D. and Albertson, C. W., "Aircraft High-Lift Aerodynamic Analysis Using a Surface-Vorticity Solver," $54 t h$ AIAA Aerospace Sciences Meeting, AIAA, San Diego, CA, 2016. 